\title{
On The Bayesian Analysis of Censored Mixture of Two Topp-Leone Distribution
}

\author{
Tabassum Naz Sindhu ${ }^{1}$, ${ }^{*}$ Zawar Hussain ${ }^{1}$ and Muhammad \\ Aslam $^{2}$ \\ ${ }^{1}$ Department of Statistics, Quaid-i-Azam University 45320 \\ Islamabad 44000, Pakistan. \\ ${ }^{2}$ Riphah International University Islamabad \\ *Corresponding Author: sindhuqau@gmail.com
}

Received: 24 $4^{\text {th }}$-January 2016 / Revised: $16^{\text {th }}$ August 2018 / Accepted: $20^{\text {th }}$ May 2019

(CIAppStat-SL2018

\begin{abstract}
This paper develops a Bayesian analysis in the context of non-informative priors for the shape parameter of the mixture of Topp-Leone using the censored data. A population of certain objects is assumed to be composed of two subgroups mixed together in an unknown proportion. The random observation taken from this population is supposed to be characterized by one of the two distinct unknown members of a Topp-Leone distribution. We model the heterogeneous population using two components mixture of the ToppLeone distribution. A comprehensive simulation scheme has been carried out to highlight the properties and behavior of the estimates in terms of sample size, corresponding risks and the mixing weights. A censored mixture data is simulated by probabilistic mixing for the computational purpose. The Bayes estimators of the said parameters have been derived under the assumption of non-informative priors using different loss functions. Posterior risks of the Bayes estimators are compared to explore the effect of prior information and loss functions. Bayes estimators assuming the uniform prior have been observed performing better.
\end{abstract}

Keywords: Mixture model, Loss function, Bayes estimators, Posterior risks, probabilistic mixing, credible intervals. 


\section{Introduction}

Topp-Leone distribution (T-L distribution) is a continuous unimodal distribution with bounded support. It is a two-parametric family continuous distribution proposed by Topp and Leone (1955). Such a distribution is useful for modeling lifetime phenomena, different aspect of this class of distributions studied by Nadarajah and Kotz (2003). The Topp-Leone distribution does not seem to be very familiar to the statisticians and has not been investigated in much detail under the Bayesian paradigm. The purpose of this study is to obtain the estimates for the parameter assuming different asymmetric loss functions.

This distribution has attracted recent attention and some key references are Ghitany et al. (2005), Van Dorp and Kotz (2004), Zhoiu et al. (2006), Kotz and Seier (2007), Nadarajah (2009), and Genç (2012). As well as having finite support, the T-L distribution has a "J-shaped" density function and a hazard function that is "bathtub-shaped". The latter characteristic is especially important in reliability applications in a wide range of fields, as is discussed recently by Reed (2011).

In recent years, the mixture models have received a considerable attention in the area of survival analysis and reliability. Mixtures of lifetime distributions occur when two different causes of failure are present, each with the same parametric form of lifetime distributions. Titterington et al. (1985), Lindsay (1995), Mclachlan and Peel (2000), Mcculloch and Searle (2001) and Demidenko (2004) are amongst the authors considering the analysis of the mixture models. The characterizations of mixtures studied by Nassar (1988), Ismail and El Khodary (2001) focused on the mixing fraction of the Exponential mixture. Further a mixture of two inverse Weibull distributions discussed by Sultan et al. (2007). Sindhu et al. (2016) analyzed Bayesian Inference of mixture of two Rayleigh distributions under double censoring. Saleem and Aslam (2008) have studied Bayesian analysis of the two component mixture of the Rayleigh distribution assuming the Uniform and the Jeffreys' prior from censored data. Saleem et al. (2010) considered the Bayesian analysis of the mixture of Power function distribution using the complete and the censored sample. Sindhu et al. (2016) have studied Bayes estimation of Gumbel mixture models with industrial applications. Sindhu et al. (2016) have evaluated the estimates of parameters by using Bayesian Approach for the Censored Shifted Gompertz Mixture Distribution. 
The article is outlined as follows. In section 2, we define the mixture model of Topp-Leone. Sampling and likelihood is developed in section 3. In sections 4, the expressions for the posterior distributions have been presented. The section 5 contains the derivation of the said estimators and corresponding posterior risks. Credible intervals are derived in sections 6. A simulation study is performed in Section 7. The real life data application is explained in Section 8. Some concluding remarks are given in section 9 .

\section{The Population and the Model}

A population is postulated to be composed of two subpopulations with specified parameters. The subpopulations are mixed in proportion $w,(1-w)$, where $0<w<1$. A finite mixture distribution function with the two component densities of specified parametric form (but with unknown parameters, $\theta_{1}$ and $\left.\theta_{2}\right)$ and with unknown mixing weights, $w$ and $(1-w)$ is: $F(x)=w F_{1}(x)+(1-w) F_{2}(x), 0<w<1$, with the two component distribution functions of specified parametric (Topp-Leone) form $F_{1}(x)=\left(2 x-x^{2}\right)^{\theta_{1}}$ and $F_{2}(x)=\left(2 x-x^{2}\right)^{\theta_{2}}$. The corresponding finite mixture density function has its probability density function (p.d.f) as:

$$
\begin{aligned}
& p\left(x \mid \theta_{1}, \theta_{2}, w\right)=w \theta_{1}(2-2 x)\left(2 x-x^{2}\right)^{\theta_{1}-1}+(1-w) \theta_{2}(2-2 x)\left(2 x-x^{2}\right)^{\theta_{2}-1}, \\
& \theta_{i}>0, i=1,2 ; 0<x<1 .
\end{aligned}
$$

\section{Sampling}

A random sample of $n$ units from the above mixture model is operating to a life testing experiment. The test is terminated at a fixed time $T$. Let the test to be conducted and it is observed that out of $n, r$ units have lifetime in the interval $[0, T]$ and $(n-r)$ units are still functioning when the test termination time $T$ is over. Hence ( $n-r)$ units that have not failed by the time $T$ are censored objects and yield no information. According to Mendenhall and Hader (1958), in many real life situations only the failed objects can easily be identified as member of either subpopulation 1 or subpopulation 2. So, depending upon the causes of failure it may be observed that $r_{1}$ and $r_{2}$ objects are identified as members of the first subpopulation and the second population respectively. Obviously $r=r_{1}+r_{2}$ and remaining (n-r) units provide no information about the subpopulation to which they belong. Furthermore, let $x_{i j}$ as the failure time of 
the $j^{\text {th }}$ unit to the $i^{\text {th }}$ subpopulation, where $j=1,2, \ldots, r_{i}, i=1,2 ; 0 \leq x_{1 j}, x_{2 j} \leq T$.

\subsection{The Maximum Likelihood Function}

The likelihood function for a two-component mixture with $\mathrm{n}$ items under study, the probability that $r_{1}$ will fail due to cause $1, r_{2}$ will fail due to cause 2 and remaining $\left(n-r_{1}-r_{2}\right)$ will survive at time $\mathrm{T}$ when test is terminated is given as

$$
\begin{aligned}
& L\left(\theta_{1}, \theta_{2}, w \mid \mathbf{x}\right) \propto \prod_{j=1}^{r_{1}} w f_{1}\left(x_{1 j}\right) \prod_{j=1}^{r_{2}} w f_{2}\left(x_{2} j\right)\{1-F(T)\}^{n-r} \\
& \mathbf{x}=\left(\mathbf{x}_{1}, \mathbf{x}_{2}\right)=\left\{x_{11}, x_{12}, \ldots, x_{1 r_{1}}, x_{21}, x_{22}, \ldots, x_{2 r_{2}}\right\} \text { is data. } \\
& L\left(\theta_{1}, \theta_{2}, w \mid \mathbf{x}\right) \propto \sum_{k_{1}=0}^{n-r} \sum_{k_{2}=0}^{n-r-k_{1}}(-1)^{k_{1}+k_{2}}\left(\begin{array}{c}
n-r \\
k_{1}
\end{array}\right)\left(\begin{array}{c}
n-r-k_{1} \\
k_{2}
\end{array}\right) \theta_{1}{ }_{1} \theta_{2}{ }^{{ }_{2}} w^{k_{1}+r_{1}}
\end{aligned}
$$

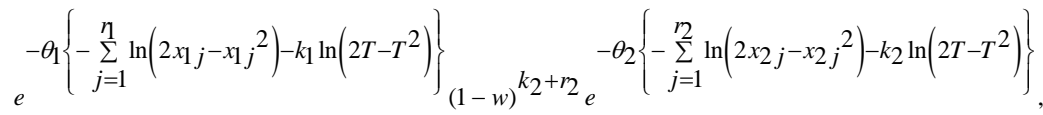

$$
\begin{aligned}
& L\left(\theta_{1}, \theta_{2}, w \mid \mathbf{x}\right) \propto \sum_{k_{1}=0}^{n-r} \sum_{k_{2}=0}^{n-r-k_{1}}(-1)^{k_{1}+k_{2}}\left(\begin{array}{c}
n-r \\
k_{1}
\end{array}\right)\left(\begin{array}{c}
n-r-k_{1} \\
k_{2}
\end{array}\right) \theta_{1}^{r_{1}} \theta_{2}^{r_{2}} w^{k_{1}+r_{1}} e^{-\theta_{1}\left\{\xi_{1 j}\left(x_{1 j}\right)\right\}} \\
& \times(1-w)^{k_{2}+r_{2}} e^{-\theta_{2}\left\{\xi_{2 j}\left(x_{2} j\right)\right\}} \text {, }
\end{aligned}
$$

where

$$
\begin{aligned}
& \xi_{1 j}\left(x_{1 j}\right)=-\left\{\sum_{j=1}^{r_{1}} \ln \left(2 x_{1 j}-x_{1 j}{ }^{2}\right)+k_{1} \ln \left(2 T-T^{2}\right)\right\} \\
& \xi_{2 j}\left(x_{2 j}\right)=-\left\{\sum_{j=1}^{r_{2}} \ln \left(2 x_{2 j}-x_{2 j}{ }^{2}\right)+k_{2} \ln \left(2 T-T^{2}\right)\right\} .
\end{aligned}
$$

\section{Prior and Posterior Distributions}

The Uniform and the Jeffreys' priors are the examples of non-informative priors which materialize the use of the Bayesian estimation methods when no prior information is available. The posterior distributions under the assumption of uniform and Jeffreys' priors have been derived and presented in the following 


\subsection{Posterior Distribution under Uniform Prior}

Let us assume a state of ignorance that $\theta_{1}, \theta_{2}$ and $w$ are uniformly distributed over $(0, \infty)$. Hence

$$
p_{1}\left(\theta_{1}\right)=k_{1}, p_{2}\left(\theta_{2}\right)=k_{2}, \theta_{i}>0 \text { and } p_{3}(w)=1,0<w<1 .
$$

Assuming independence, we have an improper joint prior that is proportional to a constant. The joint prior is incorporated with the likelihood (2) to yield a proper joint posterior distribution of $\theta_{1}, \theta_{2}$ and $w$. The joint posterior distribution of $\theta_{1}, \theta_{2}$ and $w$ is

$$
\begin{aligned}
p\left(\theta_{1}, \theta_{2}, w \mid \mathbf{x}\right) & =\frac{1}{\Omega_{1}} \sum_{k_{1}=0}^{n-r} \sum_{k_{2}=0}^{n-r-k_{1}}(-1)^{k_{1}+k_{2}}\left(\begin{array}{c}
n-r \\
k_{1}
\end{array}\right)\left(\begin{array}{c}
n-r-k_{1} \\
k_{2}
\end{array}\right) w^{r^{1}+k_{1}}(1-w)^{r_{1}+k_{1}} \\
& \times \theta_{1}^{r_{1}} \theta_{2}{ }^{r_{2}} e^{-\theta_{1}\left\{\xi_{1 j}\left(x_{1 j}\right)\right\}} e^{-\theta_{2}\left\{\xi_{2 j}\left(x_{2} j\right)\right\}}
\end{aligned}
$$

where

$$
\Omega_{1 k}=\sum_{k_{1}=0}^{n-r} \sum_{k_{2}=0}^{n-r-k_{1}}(-1)^{k_{1}+k_{2}}\left(\begin{array}{c}
n-r \\
k_{1}
\end{array}\right)\left(\begin{array}{c}
n-r-k_{1} \\
k_{2}
\end{array}\right) B\left(\kappa_{1}, \kappa_{2}\right) \frac{\Gamma\left(r_{1}+1\right) \Gamma\left(r_{2}+1\right)}{\left\{\xi_{1 j}\left(x_{1 j}\right)\right\}^{1^{1+1}}\left\{\xi_{2 j}\left(x_{2 j}\right)\right\}^{r^{2+1}}},
$$

$\kappa_{1}=r_{1}+k_{1}+1, \kappa_{2}=r_{2}+k_{2}+1$, and $B\left(\kappa_{1}, \kappa_{2}\right)$ is standard beta function.

The marginal distribution of each parameter is obtained by integrating out nuisance parameters.

$$
\begin{aligned}
& p\left(\theta_{1} \mid \mathbf{x}\right)=\frac{1}{\Omega_{1 k}} \sum_{k_{1}=0}^{n-r} \sum_{k_{2}=0}^{n-r-k_{1}}(-1)^{k_{1}+k_{2}}\left(\begin{array}{c}
n-r \\
k_{1}
\end{array}\right)\left(\begin{array}{c}
n-r-k_{1} \\
k_{2}
\end{array}\right)\left(\frac{\Gamma\left(r_{2}+1\right)}{\left\{\xi_{2 j}\left(x_{2 j}\right)\right\}^{r_{2}+1}}\right) B\left(\kappa_{1}, \kappa_{2}\right) \theta_{1}^{r_{1}} e^{-\theta_{1}\left\{\xi_{1 j}\left(x_{1 j}\right)\right\}} . \\
& p\left(\theta_{2} \mid \mathbf{x}\right)=\frac{1}{\Omega_{1 k}} \sum_{k_{1}=0}^{n-r} \sum_{k_{2}=0}^{n-r-k_{1}}(-1)^{k_{1}+k_{2}}\left(\begin{array}{c}
n-r \\
k_{1}
\end{array}\right)\left(\begin{array}{c}
n-r-k_{1} \\
k_{2}
\end{array}\right) \frac{\Gamma\left(r_{1}+1\right)}{\left\{\xi_{1 j}\left(x_{1 j}\right)\right\}^{\eta^{+1}+1}} B\left(\kappa_{1}, \kappa_{2}\right) \theta_{2}{ }^{r_{2}} e^{-\theta_{2}\left\{\xi_{2 j}\left(x_{2 j}\right)\right\}} \\
& p(w \mid \mathbf{x})=\frac{1}{\Omega_{1 k}} \sum_{k_{1}=0}^{n-r} \sum_{k_{2}=0}^{n-r-k_{1}}(-1)^{k_{1}+k_{2}}\left(\begin{array}{c}
n-r \\
k_{1}
\end{array}\right)\left(\begin{array}{c}
n-r-k_{1} \\
k_{2}
\end{array}\right) \frac{\Gamma\left(\eta_{1}+1\right) \Gamma\left(r_{2}+1\right)}{\left\{\xi_{1 j}\left(x_{1 j}\right)\right\}^{\eta+1}\left\{\xi_{2 j}\left(x_{2 j}\right)\right\}^{r_{2}+1}} w^{r_{1}+k_{1}}(1-w)^{\eta_{1}+k_{1}}
\end{aligned}
$$

\subsection{Posterior distribution under Jeffreys' prior}

Jeffreys' prior is locally uniform and hence non-informative. An appealing property of Jeffreys' prior is that it is invariant with respect to one-to-one transformations. For the Topp-Leone model given in Section 2, the Jeffreys' priors $\quad p_{1}\left(\theta_{1}\right)=\theta_{1}^{-1}, 0<\theta_{1}<\infty, p_{2}\left(\theta_{2}\right)=\theta_{2}^{-1}, 0<\theta_{2}<\infty$ and $p_{3}(w)=1,0<w<1$. assuming independence, the joint prior $g\left(\theta_{1}, \theta_{2}, w\right) \propto\left(\theta_{1} \theta_{2}\right)^{-1}$ is incorporated 
with the likelihood (2) to yield a proper joint posterior distribution of $\theta_{1}, \theta_{2}$ and $w$. The joint posterior distribution under Jeffreys' prior is:

$$
\begin{gathered}
p\left(\theta_{1}, \theta_{2}, w \mid \mathbf{x}\right)=\frac{1}{\Omega_{2}} \sum_{k_{1}=0}^{n-r} \sum_{k_{2}=0}^{n-r-k_{1}}(-1)^{k_{1}+k_{2}}\left(\begin{array}{c}
n-r \\
k_{1}
\end{array}\right)\left(\begin{array}{c}
n-r-k_{1} \\
k_{2}
\end{array}\right) w^{r_{1}+k_{1}}(1-w)^{r_{1}+k_{1}} \\
\theta_{1}^{r_{1}-1} \theta_{2}{ }^{r_{2}-1} e^{-\theta_{1}\left\{\xi_{1 j}\left(x_{1 j}\right)\right\}} e^{-\theta_{2}\left\{\xi_{2 j}\left(x_{2 j}\right)\right\}} .
\end{gathered}
$$

where

$$
\Omega_{2 k}=\sum_{k_{1}=0}^{n-r} \sum_{k_{2}=0}^{n-r-k_{1}}(-1)^{k_{1}+k_{2}}\left(\begin{array}{c}
n-r \\
k_{1}
\end{array}\right)\left(\begin{array}{c}
n-r-k_{1} \\
k_{2}
\end{array}\right) B\left(\kappa_{1}, \kappa_{2}\right) \frac{\Gamma\left(r_{1}\right) \Gamma\left(r_{2}\right)}{\left\{\xi_{1 j}\left(x_{1}\right)\right\}^{r_{1}}\left\{\xi_{2 j}\left(x_{2} j\right)\right\}^{r_{2}}} .
$$

The marginal distribution of each parameter is obtained by integrating out nuisance parameters.

$$
\begin{aligned}
& p\left(\theta_{1} \mid \mathbf{x}\right)=\frac{1}{\Omega_{2 k}} \sum_{k_{1}=0}^{n-r} \sum_{k_{2}=0}^{n-r-k_{1}}(-1)^{k_{1}+k_{2}}\left(\begin{array}{c}
n-r \\
k_{1}
\end{array}\right)\left(\begin{array}{c}
n-r-k_{1} \\
k_{2}
\end{array}\right)\left(\frac{\Gamma\left(r_{2}\right)}{\left\{\xi_{2 j}\left(x_{2 j}\right)\right\}^{r_{2}}}\right) B\left(\kappa_{1}, \kappa_{2}\right) \theta_{1}^{r_{1}-1} e^{-\theta_{1}\left\{\xi_{1}\left(x_{1}\right)\right\}} . \\
& p\left(\theta_{2} \mid \mathbf{x}\right)=\frac{1}{\Omega_{2 k}} \sum_{k_{1}=0}^{n-r} \sum_{k_{2}=0}^{n-r-k_{1}}(-1)^{k_{1}+k_{2}}\left(\begin{array}{c}
n-r \\
k_{1}
\end{array}\right)\left(\begin{array}{c}
n-r-k_{1} \\
k_{2}
\end{array}\right) \frac{\Gamma\left(r_{1}\right)}{\left\{\xi_{1 j}\left(x_{1 j}\right)\right\}^{r_{1}}} B\left(\kappa_{1}, \kappa_{2}\right) \theta_{2}^{r_{2}-1} e^{-\theta_{2}\left\{\xi_{2} j\left(x_{2 j}\right)\right\}} . \\
& p(w \mid \mathbf{x})=\frac{1}{\Omega_{2 k}} \sum_{k_{1}=0}^{n-r} \sum_{k_{2}=0}^{n-r-k_{1}}(-1)^{k_{1}+k_{2}}\left(\begin{array}{c}
n-r \\
k_{1}
\end{array}\right)\left(\begin{array}{c}
n-r-k_{1} \\
k_{2}
\end{array}\right) \frac{\Gamma\left(r_{1}\right) \Gamma\left(r_{2}\right)}{\left\{\xi_{1 j}\left(x_{1 j}\right)\right\}^{\eta_{1}}\left\{\xi_{2} j\left(x_{2} j\right)\right\}^{r_{2}}} w^{\eta_{1}+k_{1}}(1-w)^{\eta_{1}+k_{1}} .
\end{aligned}
$$

\section{Bayes Estimators and Posterior Risks}

In order to select a best decision in decision theory, a suitable loss function must be specified. The preference of loss function is a difficult job, and its selection is often formed for the reasons of mathematical convenience without any particular decision problem of ongoing interest excluding cost effect. As in the risk analysis, the potentiality of undesired event and its consequences both are explored. This potentiality is usually measured by failure rate. The Bayesian approach is extensively applied to failure rate. In disastrous outcomes, it can be terrible to underestimate the potentiality of an event rather than to overestimate. This is significant when the risk level is the basis of risk reducing initiative, either by reducing the potentiality or the consequences. An inappropriately low estimate of the risk level can lead to the lack of necessary steps to reduce the risk level. Hence, it is unreasonable to use a loss function that allows the estimation of a failure probability of zero. A positive loss at the origin allows the estimation of zero and in risk analyses estimating a zero failure probability simply means that no risk is expected for further detail see 
Norstrom (1996). Norstrom (1996) has introduced precautionary loss function and is defined as $L_{1}=\left(\theta-\hat{\theta}_{p}\right)^{2} \hat{\theta}_{p}^{-1}$, where $\hat{\theta}_{p}$ is a decision rule to estimate parameter $\theta$. The Bayes estimator under P loss function is $\hat{\theta}_{p}=\sqrt{E\left(\theta^{2} \mid \mathrm{x}\right)}$ and posterior risk under $\mathrm{P}$ loss function is $\rho\left(\hat{\theta}_{p}\right)=2\left\{\sqrt{E\left(\theta^{2} \mid \mathrm{x}\right)}-E(\theta \mid \mathrm{x})\right\}$. The weighted squared error (WSE) loss function which is of concern is $L_{2}=\theta^{-1}\left(\theta-\hat{\theta}_{W S E}\right)^{2}$, the Bayes estimator under WSE loss function is $\hat{\theta}_{W S E}=\left\{E\left(\theta^{-1} \mid \mathrm{x}\right)\right\}^{-1}$ and posterior risk under WSE loss function is $\rho\left(\hat{\theta}_{W S E}\right)=E(\theta \mid \mathrm{x})-\left\{E\left(\theta^{-1} \mid \mathrm{x}\right)\right\}^{-1}$.

The Simple Asymmetric Precautionary Loss Function (APLF) is $L_{1}=\left(\theta-\hat{\theta}_{A P L F}\right)^{2} / \theta \hat{\theta}_{A P L F}$, the Bayes estimator and posterior risk under APLF loss function are given as $\hat{\theta}_{A P L F}=\sqrt{E(\theta \mid \mathrm{x}) / E\left(\theta^{-1} \mid \mathrm{x}\right)}, \rho\left(\hat{\theta}_{A P L F}\right)=2\left\{\sqrt{E(\theta \mid \mathrm{x}) E\left(\theta^{-1} \mid \mathrm{x}\right)}-1\right\}$.

The respective marginal distribution of each parameter have been used to derive the Bayes estimators and posterior risks of $\theta_{1}, \theta_{2}$ and $w$ under Precautionary loss function (PLF), weighted squared error (WSE) loss function and Asymmetric Precautionary loss function (APLF).

\subsection{The Expressions for the Bayes Estimators and Posterior Risks using}

\section{Uniform Prior under PLF}

The Bayes estimator and posterior risk for $\theta_{1}$ under weighted loss function are derived as:

The Bayes estimator and posterior risk for $\theta_{1}$ under weighted loss function are derived as:

$$
\hat{\theta}_{1}=\sqrt{\frac{1}{\Omega_{1}} \sum_{k_{1}=0}^{n-r} \sum_{k_{2}=0}^{n-r-k_{1}}(-1)^{k_{1}+k_{2}}\left(\begin{array}{c}
n-r \\
k_{1}
\end{array}\right)\left(\begin{array}{c}
n-r-k_{1} \\
k_{2}
\end{array}\right) \frac{B\left(k_{1}, \kappa_{2}\right) \Gamma\left(r_{1}+3\right) \Gamma\left(r_{2}+1\right)}{\left\{\xi_{1 j}\left(x_{1 j}\right)\right\}^{n_{1}+3}\left\{\xi_{2 j}\left(x_{2 j}\right)\right\}^{n^{2+1}}}},
$$




$$
\rho\left(\hat{\theta}_{1}\right)=2\left\{\begin{array}{l}
\sqrt{\frac{1}{\Omega_{1}} \sum_{k_{1}=0}^{n-r} \sum_{k_{2}=0}^{n-r-k_{1}}(-1)^{k_{1}+k_{2}}\left(\begin{array}{c}
n-r \\
k_{1}
\end{array}\right)\left(\begin{array}{c}
n-r-k_{1} \\
k_{2}
\end{array}\right) \frac{B\left(\kappa_{1}, \kappa_{2}\right) \Gamma\left(r_{1}+3\right) \Gamma\left(r_{2}+1\right)}{\left\{\xi_{1 j}\left(x_{1 j}\right)\right\}^{r_{1}+3}\left\{\xi_{2 j}\left(x_{2 j}\right)\right\}^{r_{2}+1}}} \\
-\frac{1}{\Omega_{1}} \sum_{k_{1}=0}^{n-r} \sum_{k_{2}=0}^{n-r-k_{1}}(-1)^{k_{1}+k_{2}}\left(\begin{array}{c}
n-r \\
k_{1}
\end{array}\right)\left(\begin{array}{c}
n-r-k_{1} \\
k_{2}
\end{array}\right) \frac{B\left(\kappa_{1}, \kappa_{2}\right) \Gamma\left(r_{1}+2\right) \Gamma\left(r_{2}+1\right)}{\left\{\xi_{1 j}\left(x_{1 j}\right)\right\}^{r_{1}+2}\left\{\xi_{2 j}\left(x_{2 j}\right)\right\}^{r_{2}+1}}
\end{array}\right\} .
$$

The Bayes estimator and posterior risk for $\theta_{2}$ under weighted loss function are derived as:

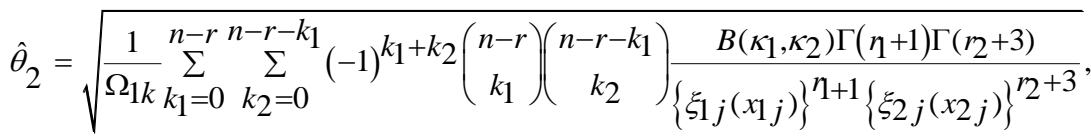

$$
\begin{aligned}
& \rho\left(\hat{\theta}_{2}\right)=2\left\{\begin{array}{l}
\sqrt{\frac{1}{\Omega_{1 k}} \sum_{k_{1}=0}^{n-r} \sum_{k_{2}=0}^{n-r-k_{1}}(-1)^{k_{1}+k_{2}}\left(\begin{array}{c}
n-r \\
k_{1}
\end{array}\right)\left(\begin{array}{c}
n-r-k_{1} \\
k_{2}
\end{array}\right) \frac{B\left(\kappa_{1}, \kappa_{2}\right) \Gamma\left(r_{1}+1\right) \Gamma\left(r_{2}+3\right)}{\left\{\xi_{1 j}\left(x_{1 j}\right)\right\}^{r_{1}+1}\left\{\xi_{2 j}\left(x_{2 j}\right)\right\}^{r^{2}+3}}} \\
-\frac{1}{\Omega_{1}} \sum_{k_{1}=0}^{n-r} \sum_{k_{2}=0}^{n-r-k_{1}}(-1)^{k_{1}+k_{2}}\left(\begin{array}{c}
n-r \\
k_{1}
\end{array}\right)\left(\begin{array}{c}
n-r-k_{1} \\
k_{2}
\end{array}\right) \frac{B\left(k_{1}, \kappa_{2}\right) \Gamma\left(r_{1}+1\right) \Gamma\left(r_{2}+2\right)}{\left\{\xi_{1 j}\left(x_{1 j}\right)\right\}^{r_{1}+1}\left\{\xi_{2 j}\left(x_{2 j}\right)\right\}^{\eta_{2}+2}}
\end{array}\right\} .
\end{aligned}
$$

The Bayes estimator and posterior risk for $w$ under weighted loss function are presented as:

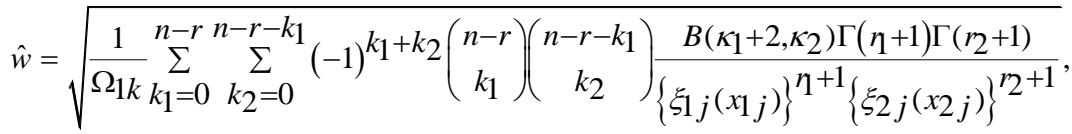

$$
\begin{aligned}
& \rho(\hat{w})=2\left\{\begin{array}{l}
\sqrt{\frac{1}{\Omega_{1}} \sum_{k_{1}=0}^{n-r} \sum_{k_{2}=0}^{n-r-k_{1}}(-1)^{k_{1}+k_{2}}\left(\begin{array}{c}
n-r \\
k_{1}
\end{array}\right)\left(\begin{array}{c}
n-r-k_{1} \\
k_{2}
\end{array}\right) \frac{B\left(\kappa_{1}+2, \kappa_{2}\right) \Gamma\left(r_{1}+1\right) \Gamma\left(r_{2}+1\right)}{\left\{\xi_{1 j}\left(x_{1 j}\right)\right\}^{\eta_{1}+1}\left\{\xi_{2 j}\left(x_{2 j}\right)\right\}^{r_{2}+1}}} \\
-\frac{1}{\Omega_{1}} \sum_{k_{1}=0}^{n-r} \sum_{k_{2}=0}^{n-r-k_{1}}(-1)^{k_{1}+k_{2}}\left(\begin{array}{c}
n-r \\
k_{1}
\end{array}\right)\left(\begin{array}{c}
n-r-k_{1} \\
k_{2}
\end{array}\right) \frac{B\left(\kappa_{1}+1, \kappa_{2}\right) \Gamma\left(r_{1}+1\right) \Gamma\left(r_{2}+1\right)}{\left\{\xi_{1 j}\left(x_{1 j}\right)\right\}^{r_{1}+1}\left\{\xi_{2 j}\left(x_{2 j}\right)\right\}^{r_{2}+1}}
\end{array}\right\} .
\end{aligned}
$$

The expressions for Bayes estimators and posterior risks under other loss functions assuming uniform prior and Jeffreys' prior can be derived with little modifications. 


\section{Credible Interval}

According to Eberly and Casella (2003) the credible interval can be defined as:

$$
\int_{0}^{L} g(\theta \mid \mathbf{x}) d \theta=\frac{\alpha}{2}, \int_{U}^{\infty} g(\theta \mid \mathbf{x}) d \theta=\frac{\alpha}{2},
$$

where $L$ and $U$ are the lower and upper limits of the credible interval respectively and $k$ is the level of significance.

\subsection{Credible interval for $\theta_{1}, \theta_{2}$ and $w$ using uniform prior}

In Bayesian inference, a confidence interval over a single model parameter $\phi$ is simply a contiguous interval $\left\{\phi_{1}, \phi_{2}\right\}$ that contains a specified proportion of the posterior probability mass over $\phi$. There is always more than one way of choosing the bounds of the interval $\left\{\phi_{1}, \phi_{2}\right\}$ to enclose $(1-\alpha) 100 \%$ of the posterior mass. There are two main conventions for determining how to choose interval boundaries: Choose the shortest possible interval enclosing $(1-\alpha) 100 \%$ of the posterior mass. This is called a highest posterior density (HPD) confidence interval. Choose interval boundaries such that an equal amount of probability mass is contained on either side of the interval. This is called a symmetric confidence interval. In this section we have obtained the symmetric confidence interval.

The $(1-\alpha) 100 \%$ credible interval for $\theta_{1}, \theta_{2}$ and $w$ on the basis of uniform prior are:

$$
\frac{\sum_{k_{1}=0}^{n-r} \sum_{k_{2}=0}^{n-r-k_{1}}(-1)^{k_{1}+k_{2}}\left(\begin{array}{c}
n-r \\
k_{1}
\end{array}\right)\left(\begin{array}{c}
n-r-k_{1} \\
k_{2}
\end{array}\right) B\left(\kappa_{1}, \kappa_{2}\right) \frac{\Gamma\left\{r_{1}+1, L \xi_{1 j}\left(x_{1 j}\right)\right\} \Gamma\left(r_{2}+1\right)}{\left\{\xi_{1 j}\left(x_{1 j}\right)\right\}^{r_{1}+1}\left\{\xi_{2 j}\left(x_{2 j}\right)\right\}^{r^{2+1}}}}{\sum_{k_{1}=0}^{n-r} \sum_{k_{2}=0}^{n-r-k_{1}}(-1)^{k_{1}+k_{2}}\left(\begin{array}{c}
n-r \\
k_{1}
\end{array}\right)\left(\begin{array}{c}
n-r-k_{1} \\
k_{2}
\end{array}\right) B\left(\kappa_{1}, \kappa_{2}\right) \frac{\Gamma\left(\eta_{1}+1\right) \Gamma\left(r_{2}+1\right)}{\left\{\xi_{1 j}\left(x_{1 j}\right)\right\}^{r_{1}+1}\left\{\xi_{2 j}\left(x_{2 j}\right)\right\}^{2^{2+1}}}}=1-\frac{\alpha}{2},
$$




$$
\frac{\sum_{k_{1}=0}^{n-r} \sum_{k_{2}=0}^{n-r-k_{1}}(-1)^{k_{1}+k_{2}}\left(\begin{array}{c}
n-r \\
k_{1}
\end{array}\right)\left(\begin{array}{c}
n-r-k_{1} \\
k_{2}
\end{array}\right) B\left(\kappa_{1}, \kappa_{2}\right) \frac{\Gamma\left\{r_{1}+1, U \xi_{1 j}\left(x_{1 j}\right)\right\} \Gamma\left(r_{2}+1\right)}{\left\{\xi_{1 j}\left(x_{1 j}\right)\right\}^{r_{1}+1}\left\{\xi_{2 j}\left(x_{2 j}\right)\right\}^{r_{2}+1}}}{\sum_{k_{1}=0}^{n-r} \sum_{k_{2}=0}^{n-r-k_{1}}(-1)^{k_{1}+k_{2}}\left(\begin{array}{c}
n-r \\
k_{1}
\end{array}\right)\left(\begin{array}{c}
n-r-k_{1} \\
k_{2}
\end{array}\right) B\left(\kappa_{1}, \kappa_{2}\right) \frac{\Gamma\left(r_{1}+1\right) \Gamma\left(r_{2}+1\right)}{\left\{\xi_{1 j}\left(x_{1 j}\right)\right\}^{r_{1}+1}\left\{\xi_{2 j}\left(x_{2 j}\right)\right\}^{r_{2}+1}}}=\frac{\alpha}{2} .
$$

$$
\frac{\sum_{k_{1}=0}^{n-r} \sum_{k_{2}=0}^{n-r-k_{1}}(-1)^{k_{1}+k_{2}}\left(\begin{array}{c}
n-r \\
k_{1}
\end{array}\right)\left(\begin{array}{c}
n-r-k_{1} \\
k_{2}
\end{array}\right) B\left(\kappa_{1}, \kappa_{2}\right) \frac{\Gamma\left(r_{1}+1\right) \Gamma\left\{r_{2}+1, L \xi_{2 j}\left(x_{2} j\right)\right\}}{\left\{\xi_{1 j}\left(x_{1 j}\right)\right\}^{r_{1}+1}\left\{\xi_{2 j}\left(x_{2 j}\right)\right\}^{r^{2}+1}}}{\sum_{k_{1}=0}^{n-r} \sum_{k_{2}=0}^{n-r-k_{1}}(-1)^{k_{1}+k_{2}}\left(\begin{array}{c}
n-r \\
k_{1}
\end{array}\right)\left(\begin{array}{c}
n-r-k_{1} \\
k_{2}
\end{array}\right) B\left(\kappa_{1}, \kappa_{2}\right) \frac{\Gamma\left(\eta_{1}+1\right) \Gamma\left(r_{2}+1\right)}{\left\{\xi_{1 j}\left(x_{1 j}\right)\right\}^{r_{1}+1}\left\{\xi_{2 j}\left(x_{2 j}\right)\right\}^{r_{2}+1}}}=1-\frac{\alpha}{2},
$$

$$
\frac{\sum_{k_{1}=0}^{n-r} \sum_{k_{2}=0}^{n-r-k_{1}}(-1)^{k_{1}+k_{2}}\left(\begin{array}{c}
n-r \\
k_{1}
\end{array}\right)\left(\begin{array}{c}
n-r-k_{1} \\
k_{2}
\end{array}\right) B\left(\kappa_{1}, \kappa_{2}\right) \frac{\Gamma\left(r_{1}+1\right) \Gamma\left\{r_{2}+1, U \xi_{2 j}\left(x_{2} j\right)\right\}}{\left\{\xi_{1 j}\left(x_{1 j}\right)\right\}^{r_{1}+1}\left\{\xi_{2 j}\left(x_{2} j\right)\right\}^{\eta^{2+1}}}}{\sum_{k_{1}=0}^{n-r} \sum_{k_{2}=0}^{n-r-k_{1}}(-1)^{k_{1}+k_{2}}\left(\begin{array}{c}
n-r \\
k_{1}
\end{array}\right)\left(\begin{array}{c}
n-r-k_{1} \\
k_{2}
\end{array}\right) B\left(\kappa_{1}, \kappa_{2}\right) \frac{\Gamma\left(r_{1}+1\right) \Gamma\left(r_{2}+1\right)}{\left\{\xi_{1 j}\left(x_{1 j}\right)\right\}^{\eta_{1}+1}\left\{\xi_{2 j}\left(x_{2}\right)\right\}^{r_{2}+1}}}=\frac{\alpha}{2} .
$$

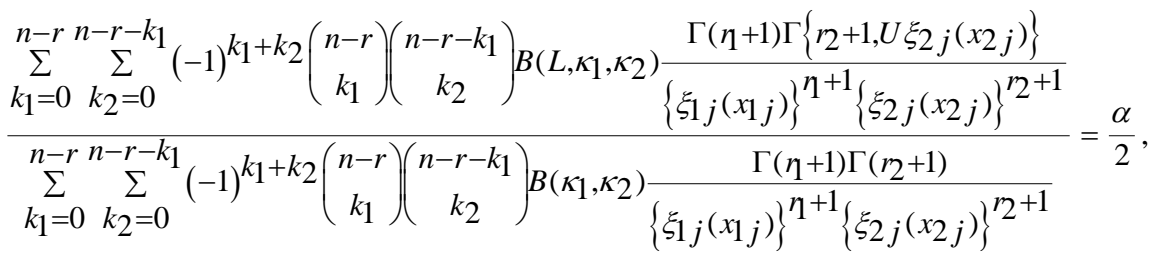

$$
\frac{\sum_{k_{1}=0}^{n-r} \sum_{k_{2}=0}^{n-r-k_{1}}(-1)^{k_{1}+k_{2}}\left(\begin{array}{c}
n-r \\
k_{1}
\end{array}\right)\left(\begin{array}{c}
n-r-k_{1} \\
k_{2}
\end{array}\right) B\left(U, \kappa_{1}, \kappa_{2}\right) \frac{\Gamma\left(r_{1}+1\right) \Gamma\left\{r_{2}+1, U \xi_{2 j}\left(x_{2} j\right)\right\}}{\left\{\xi_{1 j}\left(x_{1 j}\right)\right\}^{\eta_{1}+1}\left\{\xi_{2 j}\left(x_{2 j}\right)\right\}^{r_{2}+1}}}{\sum_{k_{1}=0}^{n-r} \sum_{k_{2}=0}^{n-r-k_{1}}(-1)^{k_{1}+k_{2}}\left(\begin{array}{c}
n-r \\
k_{1}
\end{array}\right)\left(\begin{array}{c}
n-r-k_{1} \\
k_{2}
\end{array}\right) B\left(\kappa_{1}, \kappa_{2}\right) \frac{\Gamma\left(r_{1}+1\right) \Gamma\left(r_{2}+1\right)}{\left\{\xi_{1 j}\left(x_{1 j}\right)\right\}^{r_{1}+1}\left\{\xi_{2 j}\left(x_{2 j}\right)\right\}^{n_{2}+1}}}=1-\frac{\alpha}{2} .
$$

The credible interval for the Jeffreys' prior can be constructed similarly. 


\section{Simulation Study}

A simulation study is carried out to investigate the performance of Bayes estimators and the impact of sample size and mixing proportion. We take random samples of sizes $n=50,100,200,300$ and 500 from the two component mixture of Topp-Leone distribution with $\left(\theta_{1}, \theta_{2}\right)=(3,5), w=0.3,0.6$ and censoring rate is assumed to be $20 \%$. To generate a mixture data we make use of probabilistic mixing with probability $w$ and $(1-w)$. A uniform number $u$ is generated $\mathrm{n}$ times and if $u<w$ the observation is taken randomly from $F_{1}$ (the Topp-Leone distribution with parameter $\theta_{1}$ ) otherwise from $F_{2}$ (from the Topp-Leone distribution with parameter $\theta_{2}$ ). Hence the parameters to be estimated are known to be $\theta_{1}, \theta_{2}$ and $w$.To implement censored sampling, all the observations greater than $T$ are declared as censored ones. To avoid an extreme sample, we simulate 1000 data sets each of size $n$. The Bayes estimates and posterior risks are computed using Mathematica 7.0. The average of these estimates and corresponding risks are reported in Tables 1 to 6 . Credible intervals are presented in Table 7 to 8 . The comparison observed has been summarized in last section.

Table 1: Bayes estimates (Uniform) of Topp-Leone mixture parameters and their risks (in parenthesis) with $\theta_{1}=3, \theta_{2}=5, w=0.3,0.6$ under PLF.

\begin{tabular}{|c|c|c|c|c|c|c|}
\hline$n$ & $\theta_{1}=3$ & $\theta_{2}=5$ & $w=0.3$ & $\theta_{1}=3$ & $\theta_{2}=5$ & $w=0.6$ \\
\hline \multirow{2}{*}{50} & 3.53598 & 5.35809 & 0.31416 & 3.27551 & 5.68665 & 0.59995 \\
& $(0.21115)$ & $(0.14581)$ & $(0.01293)$ & $(0.10317)$ & $(0.26149)$ & $(0.00760)$ \\
\hline \multirow{2}{*}{100} & 3.27258 & 5.15215 & 0.30728 & 0.12052 & 5.32565 & 0.599980 \\
& $(0.10308)$ & $(0.07189)$ & $(0.00672)$ & $(0.05054)$ & $(0.12757)$ & $(0.00389)$ \\
\hline \multirow{2}{*}{200} & 3.12983 & 5.09179 & 0.30370 & 3.05888 & 5.15502 & 0.599997 \\
& $(0.05069$ & $(0.03592)$ & $(0.00343)$ & $(0.02512)$ & $(0.06306)$ & $(0.00197)$ \\
\hline \multirow{2}{*}{300} & 3.08850 & 5.06285 & 0.30248 & 3.04308 & 5.09553 & 0.599999 \\
& $(0.03366)$ & $(0.02391)$ & $(0.00230)$ & $(0.01674)$ & $(0.04185)$ & $(0.00132)$ \\
\hline \multirow{2}{*}{500} & 3.05537 & 5.03531 & 0.30149 & 3.02314 & 5.060800 & 0.600150 \\
& $(0.02013)$ & $(0.01432)$ & $(0.00139)$ & $(0.01002)$ & $(0.02509)$ & $(0.00080)$ \\
\hline
\end{tabular}


Table 2: Bayes estimates (Jeffreys') of Topp-Leone mixture parameters and their risks (in parenthesis) with $\theta_{1}=3, \theta_{2}=5, w=0.3,0.6$ under PLF.

\begin{tabular}{|c|c|c|c|c|c|c|}
\hline$n$ & $\theta_{1}=3$ & $\theta_{2}=5$ & $w=0.3$ & $\theta_{1}=3$ & $\theta_{2}=5$ & $w=0.6$ \\
\hline 50 & 3.31592 & 5.22647 & 0.31416 & 3.15290 & 5.40327 & 0.59995 \\
& $(0.21059)$ & $(0.14620)$ & $(0.01293)$ & $(0.10254)$ & $(0.26043)$ & $(0.00759)$ \\
\hline \multirow{2}{*}{100} & 3.13790 & 5.08957 & 0.30728 & 3.07081 & 5.18784 & 0.59978 \\
& $(0.10205)$ & $(0.07188)$ & $(0.00672)$ & $(0.05043)$ & $(0.12731)$ & $(0.00389)$ \\
\hline \multirow{2}{*}{200} & 3.06642 & 5.05354 & 0.30370 & 3.03221 & 5.08691 & 0.599997 \\
& $(0.05048)$ & $(0.03591)$ & $(0.00342)$ & $(0.02511)$ & $(0.06299)$ & $(0.00197)$ \\
\hline \multirow{2}{*}{300} & 3.05353 & 5.02684 & 0.30248 & 3.02099 & 5.05340 & 0.599999 \\
& $(0.03365)$ & $(0.02385)$ & $(0.00231)$ & $(0.01671)$ & $(0.04185)$ & $(0.00132)$ \\
\hline \multirow{2}{*}{500} & 3.02779 & 5.01600 & 0.30149 & 3.01104 & 5.03523 & 0.600150 \\
& $(0.02009)$ & $(0.01430)$ & $(0.00138)$ & $(0.01001)$ & $(0.02508)$ & $(0.00079)$ \\
\hline
\end{tabular}

Table 3: Bayes estimates (Uniform) of Topp-Leone mixture parameters and their risks (in parenthesis) with $\theta_{1}=3, \theta_{2}=5, w=0.3,0.6$ under WSELF.

\begin{tabular}{|c|c|c|c|c|c|c|}
\hline$n$ & $\theta_{1}=3$ & $\theta_{2}=5$ & $w=0.3$ & $\theta_{1}=3$ & $\theta_{2}=5$ & $w=0.6$ \\
\hline \multirow{2}{*}{50} & 3.22835 & 5.11037 & 0.29412 & 3.09415 & 5.24361 & 0.58823 \\
& $(0.21522)$ & $(0.14601)$ & $(0.01357)$ & $(0.10324)$ & $(0.26218)$ & $(0.00792)$ \\
\hline \multirow{2}{*}{100} & 3.10240 & 5.08957 & 0.29703 & 3.06030 & 5.10605 & 0.59406 \\
& $(0.10341)$ & $(0.07265)$ & $(0.00689)$ & $(0.05101)$ & $(0.12765)$ & $(0.00398)$ \\
\hline \multirow{2}{*}{200} & 3.05100 & 5.03607 & 0.29851 & 3.01544 & 5.05374 & 0.597015 \\
& $(0.05085)$ & $(0.03597)$ & $(0.00347)$ & $(0.02513)$ & $(0.06317)$ & $(0.00199)$ \\
\hline \multirow{2}{*}{300} & 3.03557 & 5.02956 & 0.29903 & 3.01515 & 5.04516 & 0.59801 \\
& $(0.03373)$ & $(0.02393)$ & $(0.00232)$ & $(0.01675)$ & $(0.04204)$ & $(0.00133)$ \\
\hline \multirow{2}{*}{500} & 3.01409 & 5.01302 & 0.29940 & 3.01019 & 5.02245 & 0.598802 \\
& $(0.02008)$ & $(0.01432)$ & $(0.00139)$ & $(0.01003)$ & $(0.02511)$ & $(0.00079)$ \\
\hline
\end{tabular}

Table 4: Bayes estimates (Jeffreys') of Topp-Leone mixture parameters and their risks (in parenthesis) with $\theta_{1}=3, \theta_{2}=5, w=0.3,0.6$ under WSELF.

\begin{tabular}{|l|l|l|l|l|l|l|}
\hline$n$ & $\theta_{1}=3$ & $\theta_{2}=5$ & $w=0.3$ & $\theta_{1}=3$ & $\theta_{2}=5$ & $w=0.6$ \\
\hline
\end{tabular}




\begin{tabular}{|c|c|c|c|c|c|c|}
\hline 50 & $\begin{array}{l}3.01417 \\
(0.21529)\end{array}$ & $\begin{array}{l}5.02318 \\
(0.14774)\end{array}$ & $\begin{array}{l}0.29412 \\
(0.01357)\end{array}$ & $\begin{array}{l}3.01173 \\
(0.10385)\end{array}$ & $\begin{array}{l}5.02708 \\
(0.26458)\end{array}$ & $\begin{array}{l}0.588235 \\
(0.00791)\end{array}$ \\
\hline 100 & $\begin{array}{l}3.01257 \\
(0.10388)\end{array}$ & $\begin{array}{l}5.01898 \\
(0.07274)\end{array}$ & $\begin{array}{l}0.29703 \\
(0.00689)\end{array}$ & $\begin{array}{l}3.00875 \\
(0.05099)\end{array}$ & $\begin{array}{l}5.02616 \\
(0.12888)\end{array}$ & $\begin{array}{l}0.59406 \\
(0.00398)\end{array}$ \\
\hline 200 & $\begin{array}{l}3.00863 \\
(0.05099)\end{array}$ & $\begin{array}{l}5.01653 \\
(0.03609)\end{array}$ & $\begin{array}{l}0.29857 \\
(0.00347)\end{array}$ & $\begin{array}{l}3.00337 \\
(0.02524)\end{array}$ & $\begin{array}{l}5.01349 \\
(0.06346) \\
\end{array}$ & $\begin{array}{l}0.597015 \\
(0.00199) \\
\end{array}$ \\
\hline 300 & $\begin{array}{l}3.00581 \\
(0.03377)\end{array}$ & $\begin{array}{l}5.00395 \\
(0.02394)\end{array}$ & $\begin{array}{l}0.29903 \\
(0.00232)\end{array}$ & $\begin{array}{l}3.00244 \\
(0.01677)\end{array}$ & $\begin{array}{l}5.00425 \\
(0.04205)\end{array}$ & $\begin{array}{l}0.59801 \\
(0.00133)\end{array}$ \\
\hline 500 & $\begin{array}{l}3.00198 \\
(0.02014)\end{array}$ & $\begin{array}{l}5.00261 \\
(0.01433)\end{array}$ & $\begin{array}{l}0.29940 \\
(0.00139)\end{array}$ & $\begin{array}{l}3.00062 \\
(0.01004)\end{array}$ & $\begin{array}{l}5.00118 \\
(0.02513) \\
\end{array}$ & $\begin{array}{l}0.598802 \\
(0.00078) \\
\end{array}$ \\
\hline
\end{tabular}

Table 5: Bayes estimates (Uniform) of Topp-Leone mixture parameters and their risks (in parenthesis) with $\theta_{1}=3, \theta_{2}=5, w=0.3,0.6$ under APLF.

\begin{tabular}{|c|c|c|c|c|c|c|}
\hline$n$ & $\theta_{1}=3$ & $\theta_{2}=5$ & $w=0.3$ & $\theta_{1}=3$ & $\theta_{2}=5$ & $w=0.6$ \\
\hline \multirow{2}{*}{50} & 3.32826 & 5.22448 & 0.300828 & 3.15008 & 5.36654 & 0.59218 \\
& $(0.06559)$ & $(0.02837)$ & $(0.04563)$ & $(0.03306)$ & $(0.04939)$ & $(0.01341)$ \\
\hline \multirow{2}{*}{100} & 3.15143 & 5.10078 & 0.300456 & 3.07538 & 5.20334 & 0.59604 \\
& $(0.03306)$ & $(0.01423)$ & $(0.02307)$ & $(0.01659)$ & $(0.02484)$ & $(0.00668)$ \\
\hline \multirow{2}{*}{200} & 3.08312 & 5.06213 & 0.300239 & 3.03372 & 5.09480 & 0.59801 \\
& $(0.01659)$ & $(0.00713)$ & $(0.01166)$ & $(0.00831)$ & $(0.01246)$ & $(0.00333)$ \\
\hline \multirow{2}{*}{300} & 3.05319 & 5.03914 & 0.300162 & 3.02326 & 5.06213 & 0.59867 \\
& $(0.01108)$ & $(0.00475)$ & $(0.00774)$ & $(0.00554)$ & $(0.00831)$ & $(0.00222)$ \\
\hline \multirow{2}{*}{500} & 3.02416 & 5.02537 & 0.300098 & 3.01765 & 5.04047 & $\begin{array}{c}0.59920 \\
\end{array}$ \\
$(0.00665)$ & $(0.00285)$ & $(0.00465)$ & $(0.00333)$ & $(0.00499)$ & $(0.00133)$ \\
\hline
\end{tabular}

Table 6: Bayes estimates (Jeffreys') of Topp-Leone mixture parameters and their risks (in parenthesis) with $\theta_{1}=3, \theta_{2}=5, w=0.3,0.6$ under APLF

\begin{tabular}{|c|c|c|c|c|c|c|}
\hline$n$ & $\theta_{1}=3$ & $\theta_{2}=5$ & $w=0.3$ & $\theta_{1}=3$ & $\theta_{2}=5$ & $w=0.6$ \\
\hline \multirow{2}{*}{50} & 3.06954 & 5.07390 & 0.30082 & 3.04954 & 5.12344 & 0.59218 \\
& $(0.07019)$ & $(0.02919)$ & $(0.04563)$ & $(0.03419)$ & $(0.05195)$ & $(0.01341)$ \\
\hline \multirow{2}{*}{100} & 3.05497 & 5.02566 & 0.300456 & 3.02820 & 5.06033 & 0.59604 \\
& $(0.03419)$ & $(0.01444)$ & $(0.02307)$ & $(0.01687)$ & $(0.02547)$ & $(0.00668)$ \\
\hline \multirow{2}{*}{200} & $\begin{array}{c}3.02917 \\
(0.01687)\end{array}$ & $\begin{array}{c}5.02553 \\
(0.00718)\end{array}$ & $\begin{array}{c}0.30023 \\
(0.01166)\end{array}$ & $\begin{array}{c}3.01050 \\
(0.00838)\end{array}$ & $\begin{array}{c}5.04487 \\
(0.01261)\end{array}$ & $\begin{array}{c}0.598012 \\
(0.00333)\end{array}$ \\
\hline \multirow{2}{*}{300} & $\begin{array}{c}3.01694 \\
(0.01120)\end{array}$ & $\begin{array}{c}5.01056 \\
(0.00477)\end{array}$ & $\begin{array}{c}0.300162 \\
(0.00774)\end{array}$ & $\begin{array}{c}3.00683 \\
(0.00557)\end{array}$ & $\begin{array}{c}5.01953 \\
(0.00838)\end{array}$ & $\begin{array}{c}0.598672 \\
(0.00222)\end{array}$ \\
\hline \multirow{2}{*}{500} & $\begin{array}{c}3.01424 \\
(0.00670)\end{array}$ & $\begin{array}{c}5.00973 \\
(0.00286)\end{array}$ & $\begin{array}{c}0.30009 \\
(0.00465)\end{array}$ & $\begin{array}{c}3.00514 \\
(0.00334)\end{array}$ & $\begin{array}{c}5.00865 \\
(0.00501)\end{array}$ & $\begin{array}{c}0.59920 \\
(0.00133)\end{array}$ \\
\hline
\end{tabular}


Table 7: The lower limit (LL), upper limit (UL) 95\% credible intervals under uniform prior

\begin{tabular}{|l|c|c|c|c|c|c|}
\hline \multirow{2}{*}{$n$} & \multicolumn{2}{|c|}{$\theta_{1}=3$} & \multirow{2}{*}{ Width } & \multicolumn{2}{|c|}{$\theta_{2}=5$} & \multirow{2}{*}{ Width } \\
\cline { 2 - 5 } & LL & UL & & LL & UL & \\
\hline 50 & 2.09632 & 4.26240 & 2.16608 & 2.09760 & 7.71386 & 5.61626 \\
\hline 100 & 2.3367 & 3.86771 & 1.53101 & 2.33673 & 6.81285 & 4.47612 \\
\hline 200 & 2.51275 & 3.59110 & 1.07835 & 2.51275 & 6.23021 & 3.71746 \\
\hline 300 & 2.59573 & 3.47512 & 0.87939 & 2.59573 & 5.98983 & 3.39410 \\
\hline 500 & 2.67835 & 3.35793 & 0.67958 & 2.67835 & 5.73262 & 3.05427 \\
\hline
\end{tabular}

\begin{tabular}{|l|c|c|}
\hline \multicolumn{2}{|c|}{$w=0.6$} & \multirow{2}{*}{ Width } \\
\hline \multicolumn{1}{|c|}{ LL } & UL & \\
\hline 0.461141 & 0.724157 & 0.26302 \\
\hline 0.50174 & 0.690691 & 0.18895 \\
\hline 0.530738 & 0.665425 & 0.13469 \\
\hline 0.54358 & 0.653849 & 0.11027 \\
\hline 0.556425 & 0.642026 & 0.08560 \\
\hline
\end{tabular}

Table 8: 95\% of the lower limit (LL) and upper limit (UL) credible intervals under Jeffreys' prior

\begin{tabular}{|l|c|l|l|l|l|l|}
\hline \multirow{2}{*}{$n$} & \multicolumn{2}{|c|}{$\theta_{1}=3$} & \multirow{2}{*}{ Width } & \multicolumn{2}{|c|}{$\theta_{2}=5$} & \multirow{2}{*}{ Width } \\
\cline { 2 - 3 } \cline { 5 - 7 } & LL & UL & & LL & UL & \\
\hline 50 & 2.10450 & 4.14515 & 2.13065 & 2.01647 & 7.40980 & 5.39333 \\
\hline 100 & 2.29294 & 3.81131 & 1.51837 & 2.29298 & 6.66846 & 4.37548 \\
\hline 200 & 2.48996 & 3.56384 & 1.07388 & 2.48996 & 6.16080 & 3.67084 \\
\hline 300 & 2.58026 & 3.45722 & 0.87696 & 2.58026 & 5.94436 & 3.36410 \\
\hline 500 & 2.66982 & 3.34737 & 0.67845 & 2.66892 & 5.70593 & 3.03701 \\
\hline
\end{tabular}

\begin{tabular}{|l|c|l|}
\hline \multicolumn{2}{|c|}{$w=0.6$} & \multirow{2}{*}{ Width } \\
\cline { 1 - 2 } \multicolumn{1}{|c|}{ LL } & UL & \\
\hline 0.461141 & 0.724157 & 0.26302 \\
\hline 0.50174 & 0.690691 & 0.18895 \\
\hline 0.530738 & 0.665425 & 0.13469 \\
\hline 0.54358 & 0.653849 & 0.11027 \\
\hline 0.556425 & 0.642026 & 0.08560 \\
\hline
\end{tabular}




\section{Real Data Example}

This section includes the analysis of a real life data to illustrate the methodology discussed in the previous section. In order to discuss the practical applicability of the results obtained under above sections, the following real life data on Survival times for 30 light bulbs in months presented by Butler (2011) have been used for analysis. The idea has been to verify whether the outcomes and properties of the Bayes estimators explored by simulation study have the same behavior under a real life environment. It is assumed that mixing weight and censoring rate are $w=0.6$ and $T=0.6$, respectively. Under these assumptions, the distribution of observed data into censored subsamples of size $r_{1}=16$ and $r_{2}=12$, respectively. The Bayes estimates under type I mixture censored samples based on uniform and Jeffreys' prior using PLF, WSELF and APLF have been presented in the following tables.

Table 9: Bayes estimates (BE) and posterior risks (PR) of Topp-Leone mixture using the UP and JP under PLF, WSELF and APLF with real life mixture data.

\begin{tabular}{|c|c|c|c|c|c|}
\hline $\begin{array}{c}\text { Loss } \\
\text { Function }\end{array}$ & \multicolumn{2}{|c|}{ Prior } & $\hat{\theta}_{1}$ & $\hat{\theta}_{2}$ & $\hat{w}$ \\
\hline \multirow{4}{*}{ PLF } & \multirow{2}{*}{ UP } & $\mathrm{BE}$ & 4.33437 & 0.60929 & 0.59323 \\
\hline & & PR & 0.22382 & 0.04422 & 0.01274 \\
\hline & \multirow{2}{*}{ JP } & $\mathrm{BE}$ & 4.11027 & 0.56461 & 0.59341 \\
\hline & & PR & 0.22397 & 0.04491 & 0.01276 \\
\hline \multirow{4}{*}{ WSELF } & \multirow{2}{*}{ UP } & $\mathrm{BE}$ & 3.55548 & 0.54212 & 0.57313 \\
\hline & & PR & 0.22697 & 0.04507 & 0.01371 \\
\hline & \multirow{2}{*}{ JP } & $\mathrm{BE}$ & 3.77137 & 0.49741 & 0.57333 \\
\hline & & PR & 0.22704 & 0.09451 & 0.01397 \\
\hline \multirow{4}{*}{ APLF } & \multirow{2}{*}{ UP } & $\mathrm{BE}$ & 4.10743 & 0.56420 & 0.57995 \\
\hline & & PR & 0.05602 & 0.03147 & 0.02378 \\
\hline & \multirow{2}{*}{ JP } & $\mathrm{BE}$ & 3.88324 & 0.51947 & 0.58014 \\
\hline & & PR & 0.05932 & 0.08872 & 0.01248 \\
\hline
\end{tabular}

\section{Conclusion}

Tables 1-8 appended above give Bayes estimates, corresponding risks, and 95 $\%$ credible intervals for the parameters of the mixture model. Which are empirically evaluated based on a Monte Carlo simulation study of samples. 
This simulation study has displayed some interesting properties of the Bayes estimates. The Bayes estimates of mixing proportion parameter ' $w$ ' assuming the Uniform and Jeffreys' priors under all loss functions are identical and equally efficient.

The interesting remark concerning the posterior risks of the estimates $\theta_{1}, \theta_{2}$ and $w$ is that increasing (decreasing) the proportion of a component in the mixture reduces (increases) the posterior risk of the corresponding $\theta$ parameter's estimate. Further, the increase in sample size reduces the posterior risks of the estimates $\theta_{1}, \theta_{2}$ and $w$.The Bayes estimates of $\theta_{1}, \theta_{2}$ are overestimated, but the extent of this overestimation is inversely proportional to the sample size. On the other hand Bayes estimates of mixing proportion parameter are underestimated with few exceptions. The posterior risk of the mixing parameter $w$ is same under all loss functions assuming uniform and Jeffreys' prior. The performance of estimates under the assumption of Uniform prior is better than Jeffreys' prior under WSELF and APLF loss function. The Bayes estimates under Uniform prior are more precise than its non-informative counterparts. Also, the results, obtained through real life data reported in Table 9 coincide with the simulation results with few exceptions showing the correctness of the simulation scheme.

Tables 7-8, give the results of Interval estimation. The credible intervals work quite well under the Jeffreys' prior. The width of credible interval is inversely proportional to sample size. The findings of the present study suggest that in order to estimate $\theta_{1}, \theta_{2}$ and $w$, the use of asymmetric loss function under the uniformed prior can be preferred.

\section{References}

1. Butler E.L. (2011). Estimating the survival distribution of aluminum processing pots. Carnegie Mellon University Research Showcase.

2. Demidenko, E. (2004). Mixed Models: Theory and applications. Wiley, New Jersey

3. Eberly L.E. and Casella G. (2003). Estimating Bayesian credible intervals. Journal of Statistical Planning and Inference, 112: 115-132.

4. Genç A. I. (2012). Moments of order statistics of Topp-Leone distribution. Statistical Papers, 53: 117-131. 
5. Ghitany M., Kotz S. and Xie M. (2005). On some reliability measures and their stochastic orderings for the Topp-Leone distribution. Journal of Applied Statistics, 32: 715-722.

6. Gosh S.K. and Ebrahimi N. (2001). Bayesian Analysis of the Mixing Function in a Mixture of Two Exponential Distributions. Institute of Statistics Mimeo Series No. 2531.

7. Ismail S.A. and El-Khodary I.H. (2001). Characterization of mixtures of exponential family distributions through conditional expectation. Annual Conference on Statistics and Computer Modeling in Human and Social Sciences, 13: 64-73.

8. Kotz S. and Seier E. (2007). Kurtosis of the Topp-Leone distributions. InterStat, July, \#001.

9. Landsay, B. G. (1995). Mixture models: Theory, geometry and applications. The institute of mathematical statistical, Hayward, CA.

10. Maclachlan G. J. and Peel D. (2000). Finite mixture models. Wiley, New York.

11. Mcculloch C.E. and Searle S.R. (2001). Generalized, Linear and Mixed Models. Wiley, New York.

12. Mendenhall W. and Hadar R.J. (1958). Estimation of parameters of mixed exponential distributions from Censored Life Test Data. John Wiley and Sons.

13. Nadarajah S. and Kotz S. (2003). Moments of some J-shaped distributions. Journal of Applied Statistics, 30: 311-317.

14. Nadarajah S. (2009). Bathtub-shaped failure rate functions. Quality and Quantity, 43: 855-863.

15. Nassar M. M. (1988). Two properties of mixtures of exponential distributions. IEE Transactions on Reliability, 37(4): 383-385.

16. Norstrom, J.G., (1996). The use of precautionary loss functions in risk analysis. IEEE Transactions on reliability, 45(3), pp.400-403.

17. Reed W. J. (2011). A flexible parametric survival model which allows a bathtub shaped hazard rate function. Journal of Applied Statistics, 38: 1665-1680.

18. Saleem M. and Aslam M. (2008). Bayesian analysis of the two component mixture of the Rayleigh distribution assuming the Uniform and the Jeffreys' prior from censored data. Journal of Applied Statistical Science, 16(4): 105-113. 
19. Saleem M., Aslam M. and Economou P. (2010). On the Bayesian analysis of the mixture of Power function distribution using the complete and the censored sample. Journal of Applied Statistics, 37(1): 25-40.

20. Sindhu T.N., Aslam M. and Hussain Z. (2016). A Simulation Study of Parameters for the Censored Shifted Gompertz Mixture Distribution: A Bayesian Approach. Journal of statistics and management systems,19(3): 423-450.

21. Sindhu T. N., Feroze N., and M. Aslam (2016). Bayesian Inference of Mixture of Two Rayleigh Distributions: A New Look. Journal of Mathematics, Punjab University. 48(2) 49-64.

22. Sindhu T.N., Riaz M., Aslam M. and Ahmed Z. (2015). Bayes estimation of Gumbel mixture models with industrial applications. Transactions of the Institute of Measurement and Control, 38: 201-214.

23. Sultan K.S., Ismail A.M. and Al-Moisheer A.S. (2007). Mixture of two inverse Weibull distributions: properties and estimation. Computational Statistics and Data Analysis, 51: 5377-5387.

24. Titterington D.M., Smith A.F.M. and Makov U. E. (1985). Statistical analysis of finite mixture distributions. Wiley, London.

25. Topp C.W. and Leone F.C. (1955). A family of J-shaped frequency functions. Journal of the American Statistical Association, 50: 313-321.

26. Van D.J.R. and Kotz S. (2004). Modeling income distributions using elevated distributions on a bounded domain. Unpublished manuscript, The George Washington University, Washington DC.

27. Zhou M., Yang D.W., Wang Y. and Nadarajah S. (2006). Some J-shaped distributions: Sums, products and ratios. RAMS '06. Annual Reliability and Maintainability Symposium, 175-181 